$\xi=-1$

\title{
Application of Douglass-Gunn ADI Scheme on Diffusion Model with Different Noise Level for Image Denoising
}

\author{
Nur Aimi Abdul Aziz, Suhaila Abd Halim* \\ Faculty of Computer and Mathematical Sciences, Universiti Teknologi MARA,40450 Shah Alam, Selangor, Malaysia \\ *Corresponding author E-mail: suhaila@tmsk.uitm.edu.my
}

\begin{abstract}
Noise level is the amount of noise that corrupted the clear image in order to test on the filtering method of an algorithm proposed for image denoising. Most of the existing filtering techniques are able to remove noise but unable to preserve the image detail well and hence causing the blurring effect. Due to that, the objectives of this paper are to propose and implement Douglas-Gunn Alternating Direction Implicit (DG-ADI) on Anisotropic Diffusion (AD) model. Then, measure the performance of the proposed scheme with different level of noise. PDE based model is applied with the unconditional stable of DG-ADI scheme to remove the noise that corrupted the images. The $\mathrm{AD}$ model is used for preserving the image structures and edges. In this paper, a set of grayscale images from standard database is being filtered with three different noise levels in order to measure the performance of the proposed schemes. The performance of the proposed scheme is measured using the Mean Structural Similarity Index (MSSIM), Peak Signal to Noise Ratio (PSNR), Universal Image Quality Index (UIQI) and processing time. The implementation of the algorithm is completed using MATLAB R2013a. Experimental results show that the DG-ADI scheme able to remove noise with different noise level. The used of DG-ADI scheme in solving the AD model can remove the noise well without destroy the structure of image with appropriate parameters setting in grayscale image.
\end{abstract}

Keywords: Anisotropic Diffusion; Douglass-Gunn ADI; Image Denoising; MATLAB; Noise.

\section{Introduction}

Noise is the main contributor that leads to the loss of useful information in an image. The additive of noise usually happens during the acquisition and transmission phase. Image denoising is a process that holds a main goal which is to remove the noise efficiently. The clear image should be recovered from the disruption of additive noise. There are several types of noise being used in the previous studies such as Gaussian noise, speckle noise, Poisson noise and salt-and-pepper noise [2, 12]. Over various researches conducted, the well-known noise being used is the Gaussian noise $[5,8,10]$ since it is popular with the digital image processing since it is independent of each pixel and signal intensity. Otherwise, this type of noise has the characteristic where the noise level is constant at the dark region. Therefore, it is said suitable for the grayscale images and Gaussian noise is being used for the experimental purpose. There are several researches conducted for examining the different level of noise. In [1] introduced an algorithm that automatically detects the level of noise in input data. The Perona Malik anisotropic diffusion (PMAD) model [13] is a new scale space representation which was pioneering by [16]. The new anisotropic model is developed to overcoming the problem arises due to the blurring effect of the previous model proposed. The nonlinear diffusion functions are used as done by [15]. This paper highlight on the ability of Douglas-Gunn Alternating Direction Implicit (DG-ADI) scheme [9] in filtering the noise in grayscale images with an appropriate parameters selection. In solving the multidimensional spaces alternately, the unconditional stable ADI scheme been applied, and it may reduce the computational cost.
The parameters with a diffusion coefficient (DC) function are gradient threshold, $K$, time step, $t$ and the number of iterations. Over the years, there are lots of work has been done for image denoising by solving the Anisotropic Diffusion (AD) equation with different DC function. In [13] introduced two DC function and further modification of the Perona and Malik function $[6,14$, $17,3]$ is developed to enhance the quality of image. In this paper, the higher range of parameter is used and one type of DC function being applied to test the performance of the scheme.

The scheme performance is evaluated based on the Peak Signal to Noise Ratio (PSNR), Mean Square Error (MSE) and other quality measurement formula. In [4] evaluated the performance using PSNR and MSE and another quality evaluation technique which is SSIM to obtain a more accurate and detail results [11]. For this paper the MSSIM is used which is the average value of SSIM is taken into consideration for an optimum results. All the algorithm are solve by using MATLAB R2013a.

The objectives of this paper are to propose a discretized scheme using DG-ADI on AD model and implement the proposed scheme on a set of grayscale image. Then, the performance of the proposed scheme on different level of noise is evaluated using image quality metrics and processing time in order to measure the accuracy and the efficiency of the proposed scheme.

This paper is organized as follows: Section 2 is the methodology of the process involved. Section 3 presents the results of the image denoising for the proposed scheme with distruption three different noise levels with fixed parameters selection. Section 4 gives the conclusion of the research. 


\section{Methodology}

The application of DG-ADI schemes into AD model begin by setting up the $\mathrm{AD}$ model, follow by the discretization process. Then, the scheme is applied into the denoising process with addition of Gaussian noise on each input data using three different noise levels. The three noise levels of $0.01,0.05$ and 0.1 are being tested on five grayscale images from a standard database. The results of the denoised image are evaluated to in order to measure the efficiency of the proposed scheme. The process is conducted separately, and the implementation is also repeated for each noise level using MATLAB R2013a. Figure 1 shows the flow of the denoising process.

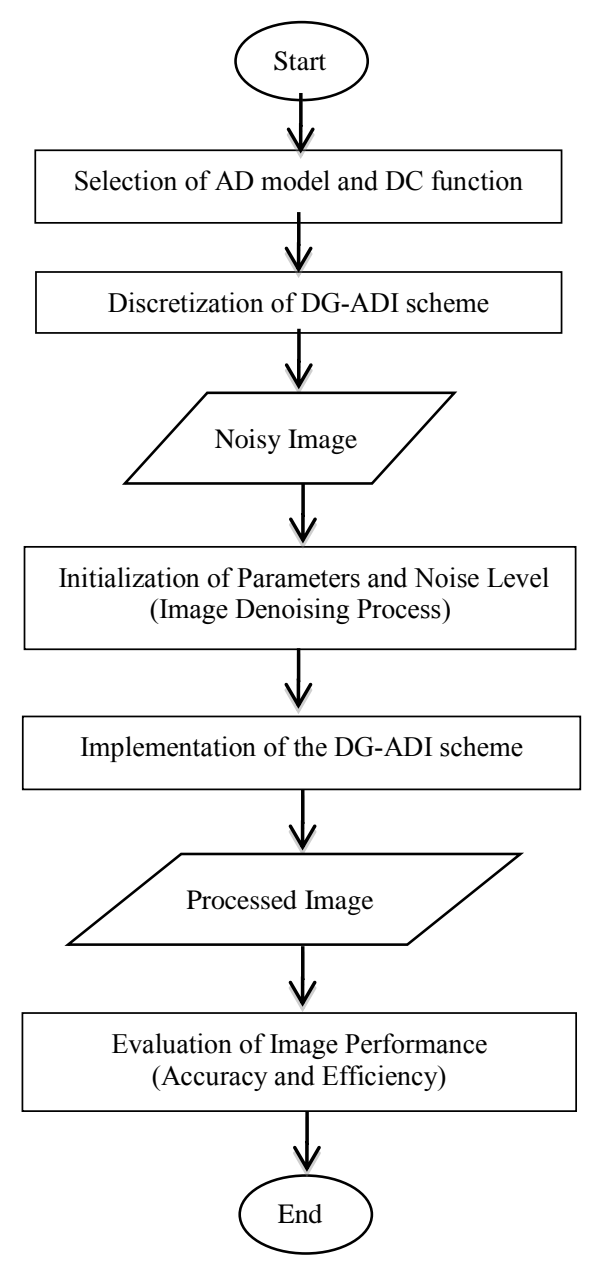

Fig. 1: Process Flow of Methodology

The AD model that was introduced by [14] controlled by several parameters. It is defined as follows:

$$
\frac{\partial I(x, y, t)}{\partial t}=\operatorname{div}(c(x, y, t) \square \nabla I(x, y, t))
$$

where $I(x, y, t)$ is denoted as the two dimensional image intensity of the current pixel coordinate, $(x, y)$ at time $t, c(x, y, t)$ is the diffusion coefficient (DC) function related to the $\mathrm{AD}$ model and div is indicate as the divergence operator. In this paper, $c(x, y, t)$ proposed by [14] is used. The DC function used is defined as:

$$
c(x, y, t)=c(\nabla I(x, y, t))=\frac{1}{1+\left(\frac{|\nabla I(x, y, t)|}{K}\right)^{2}}
$$

where $K$ is the contrast parameter that control the diffusion on image edge.

AD model is discretized using DG-ADI scheme of the finite difference method (FDM). The two dimensional model is discretized into two split schemes of DG-ADI, so that one dimensional space can be solves alternately at different time step.

By discretizing in (1), it forms in (3) with two time split schemes with the replacement of $I(x, y, t)=I_{i, j}^{n} \quad$ and $c(|\nabla I(x, y, t)|)=c\left(\left|\nabla I_{i, j}\right|\right)$.

a) $\left(1-\frac{\mu_{x} c\left\|\nabla I_{i, j}\right\|}{2} \delta_{x}^{2}\right) I_{i, j}^{*}=\left(1+\frac{\mu_{x} c\left\|\nabla I_{i, j}\right\|}{2} \delta_{x}^{2}+\mu_{y} c\left\|\nabla I_{i, j}\right\| \delta_{y}^{2}\right) I_{i, j}^{n}$

b) $\left(1-\frac{\mu_{y} c\left\|\nabla I_{i, j}\right\|}{2} \delta_{y}^{2}\right) I_{i, j}^{n+1}=I_{i, j}^{*}-\frac{\mu_{y} c\left\|\nabla I_{i, j}\right\|}{2} \delta_{y}^{2} I_{i, j}^{n}$

By introducing the intermediate level of $I_{i, j}^{n+\frac{1}{2}}$ in (3), then, it forms in (4).

a) $\left(1-\frac{\mu_{x} c\left\|\nabla I_{i, j}\right\|}{2} \delta_{x}^{2}\right) I_{i, j}^{n+\frac{1}{2}}=\left(1+\frac{\mu_{x} c\left\|\nabla I_{i, j}\right\|}{2} \delta_{x}^{2}+\mu_{y} c\left\|\nabla I_{i, j}\right\| \delta_{y}^{2}\right) I_{i, j}^{n}$
b) $\left(1-\frac{\mu_{y} c\left\|\nabla I_{i, j}\right\|}{2} \delta_{y}^{2}\right) I_{i, j}^{n+1}=I_{i, j}^{n+\frac{1}{2}}-\frac{\mu_{y} c\left\|\nabla I_{i, j}\right\|}{2} \delta_{y}^{2} I_{i, j}^{n}$

where $\mu_{x}=\frac{\Delta t}{\Delta x^{2}}, \mu_{y}=\frac{\Delta t}{\Delta y^{2}}$ and letting the time step $d x=d y=1$ forming the two fractional schemes for $n+1$ and $n+\frac{1}{2}$ time split.

The iteration is calculated to obtain the first half fractional time $n+\frac{1}{2}$ for scheme in (4a) and it iterates for the other half fractional step $n+1$ for scheme in (4b).

There are five grayscale images from standard database are being tested in this paper which are Baby, Lena, Flower, Girl and Elephant image. All the images are corrupted with addition of Gaussian noise with density $0.01,0.05$ and 0.1 .

For simulating the effects of the ADI scheme proposed, addition of noise to the images acts as the medium for solving the problem arises. The noise is added with the purpose on testing how well the algorithm when different amount of noise present. In [7] defined the equation of Gaussian noise as follows:

$\rho_{G}(z)=\frac{1}{\sigma \sqrt{2 \pi}} e^{-\frac{(z-\mu)^{2}}{2 \sigma^{2}}}$

where

$z$ : represent the gray image for Gaussian noise or white Gaussian $\sigma:$ standard deviation

$x:$ random variables

$\mu$ : mean variables

Figure 2, Figure 3 and Figure 4 show the original images that have been corrupted with $0.01,0.05$ and 0.1 Gaussian noise. The discretized scheme in (4) is used to filter the noise.
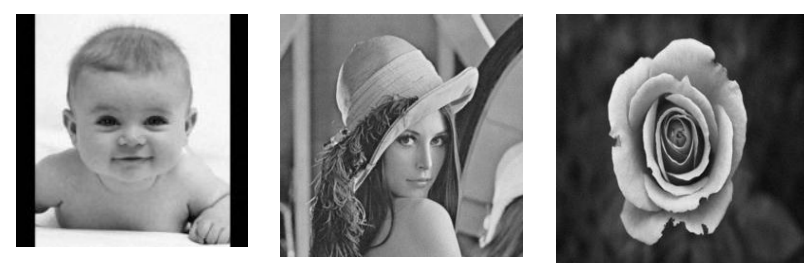


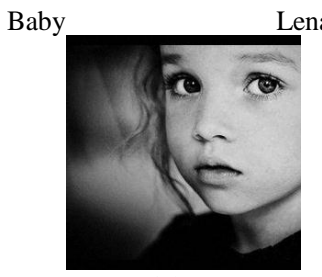

Girl

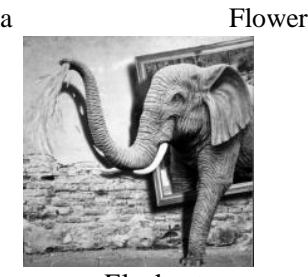

Elephant
Fig. 2: Original Image with Addition of Noise Density, $d=0.01$
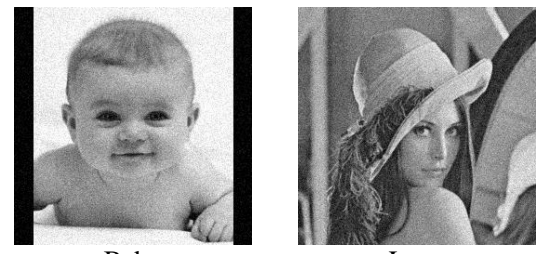
Lena

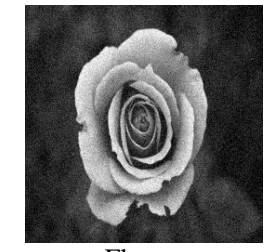

Baby

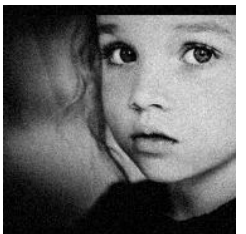

Girl

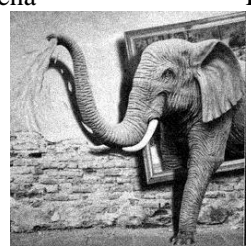

Elephant

Fig. 3: Original Image with Addition of Noise Density, $d=0.05$

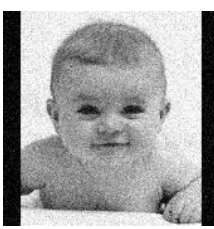

Baby

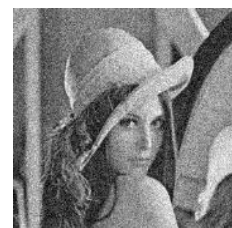

Lena

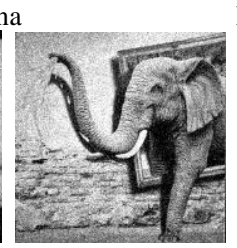

Elephant

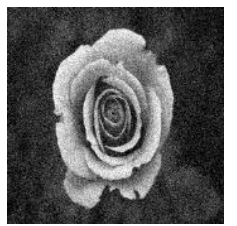

Flower

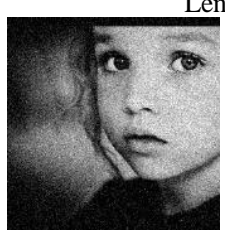

Girl
Fig. 4: Original Image with Addition of Noise Density, $d=0.1$

In order to evaluate the performance, three quality measurement approaches which are the PSNR, MSSIM, UIQI and another addition of processing time to record the efficiency of the schemes.

$$
\begin{aligned}
& P S N R=10 \log _{10}\left(\frac{255^{2}}{M S E}\right) d B \\
& M S E=\frac{1}{M N} \sum_{i=1}^{M} \sum_{j=1}^{N}\left(I_{i, j}-D_{i, j}\right)^{2} \\
& M S S I M=\frac{1}{M N} \sum_{i}^{M} \sum_{j}^{N} \frac{\left(2 \mu_{i} \mu_{j}+C_{1}\right)\left(2 \sigma_{i j}+C_{2}\right)}{\left(\mu_{i}^{2} \mu_{j}^{2}+C_{1}\right)\left(\sigma_{i}^{2} \sigma_{j}^{2}+C_{2}\right)} \\
& U I Q I=\frac{\bar{i} \bar{j}}{\left(\sigma_{i}^{2}+\sigma_{j}^{2}\right)\left((\bar{i})^{2}+(\bar{j})^{2}\right)}
\end{aligned}
$$

The processing time is the time recorded starting from the processing of the input noisy image until the implementation of the scheme is completed and produced the required processed image. Table 1 shows the algorithm for the entire process of the denoising using the proposed DG-ADI scheme.
Table 1: Algorithm for DG-ADI scheme for Noise Removal

\begin{tabular}{|l|}
\hline Input: Original image and noisy image \\
Output : Processed image \\
Initialization: $d x, d y, d=0.01,0.05,0.1$, number of iteration $=30$, time \\
step $=0.15, K=10$ \\
For I $=1:$ number of iteration \\
\hline Use DC function: equation (2) \\
\hline Application of DG-ADI Scheme: equation (4a) and (4b) \\
\hline Evaluation using $M S S I M, P S N R, U I Q I$ and processing time (PT) \\
\hline End
\end{tabular}

\section{Results and Discussion}

This section discusses on the quality of images based for three different noise density levels, $d=0.01,0.05,0.1$. The images are tested with a DC function with specific parameter estimation. 30 iterations are run for all the images and the quality of images are recorded when time reach at the highest MSSIM value. This is due to the accuracy of the MSSIM which is more accurate that the $P S N R$ and UIQI since it measures the structural information for the image quality. The quality metrics are used to measure the accuracy of the scheme. In addition, the processing time $(P T)$ is second (s) also recorded in order to measure the efficiency of the scheme. Table 2 illustrates the quality of images on the DG-ADI scheme with corresponding parameters $K=10$ and time step $=$ 0.15 .

Table 2. MSSIM, $P S N R, U I Q I$ and $P T$ for Different Noise Density Level

\begin{tabular}{|c|c|c|c|c|c|}
\hline$d$ & Image & $M S S I M$ & $P S N R$ & $U I Q I$ & $P T(s)$ \\
\hline 0.01 & Baby & 42.560032 & 0.940492 & 0.002833 & 77.98178 \\
\cline { 2 - 6 } & Lena & 39.619550 & 0.954815 & 0.003535 & 81.26297 \\
\cline { 2 - 6 } & Flower & 40.547556 & 0.962419 & 0.003523 & 80.89422 \\
\cline { 2 - 6 } & Girl & 41.853077 & 0.941322 & 0.003174 & 81.78838 \\
\cline { 2 - 6 } & Elephant & 38.731737 & 0.961144 & 0.003698 & 81.21809 \\
\hline 0.05 & Baby & 33.79265 & 0.826981 & 0.002248 & 84.1439 \\
\cline { 2 - 6 } & Lena & 30.76390 & 0.876851 & 0.002876 & 81.1263 \\
\cline { 2 - 6 } & Flower & 31.81725 & 0.921163 & 0.002971 & 81.9774 \\
\cline { 2 - 6 } & Girl & 32.96270 & 0.811223 & 0.002527 & 80.6870 \\
\cline { 2 - 6 } & Elephant & 28.00069 & 0.871133 & 0.003219 & 80.9627 \\
\hline 0.1 & Baby & 29.189978 & 0.766759 & 0.001910 & 93.7794 \\
\cline { 2 - 6 } & Lena & 27.047423 & 0.787247 & 0.002332 & 89.6155 \\
\cline { 2 - 6 } & Flower & 27.927584 & 0.869835 & 0.002550 & 94.7077 \\
\cline { 2 - 6 } & Girl & 28.911795 & 0.734147 & 0.002172 & 95.0837 \\
\cline { 2 - 6 } & Elephant & 24.103014 & 0.767146 & 0.002748 & 81.0535 \\
\hline
\end{tabular}

Table 3 shows the results of the processed images with different noise density for the five noisy images.

Table 3: Processed Image

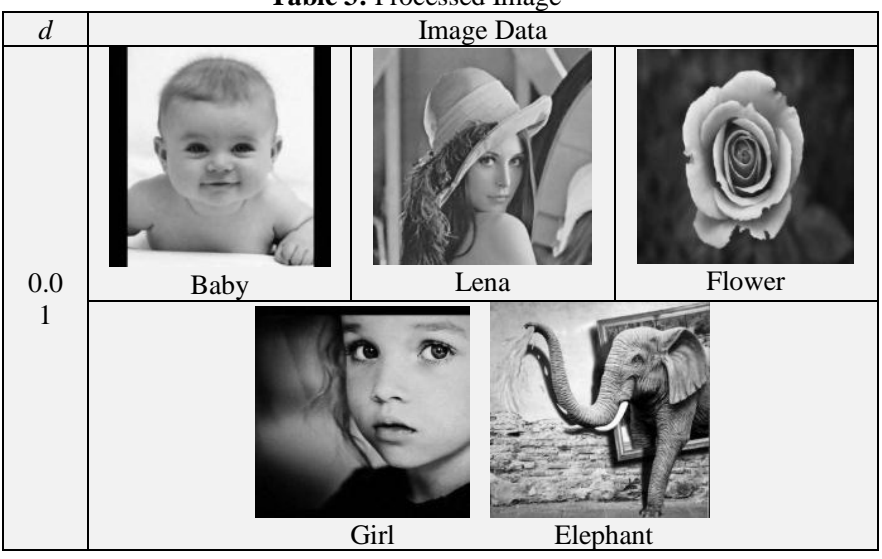




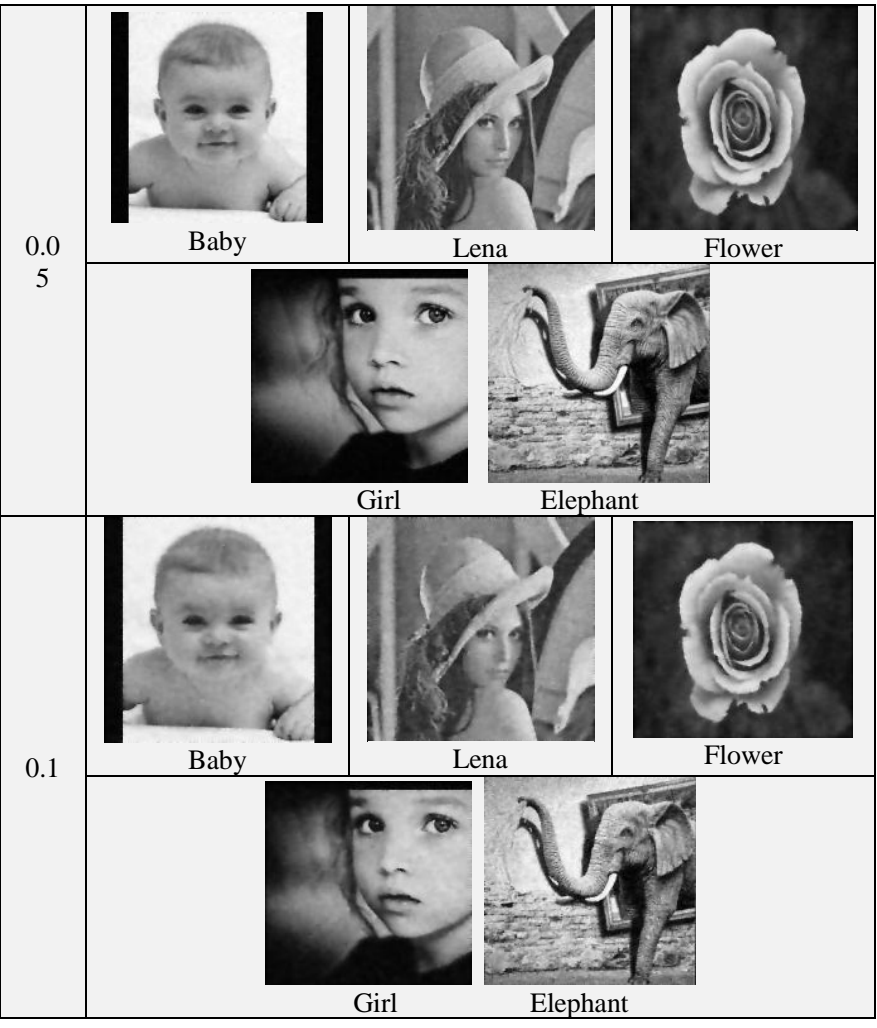

Based on the experimental results, the three level of noise are added to the image gave different quality of image. The results are measured based on the number of iterations for the maximum $M S S I M$ value. For the noise level, $d=0.01$, it shows the highest MSSIM value compared to the other noise level. The results are said to be logic since the higher level of noise, the lower the quality of image. This is due to aim in preserving the edges and maintain the fine structures from over-smoothing effects. For example, the Girl image give the highest MSSIM value for noise level, $d=$ 0.01 follow by $d=0.05$ and $d=0.1$. The PSNR and UIQI value shows in the range of high quality image and it is acceptable based on the previous literature research. There might be a blurring effect for the $d=0.1$ since the noise that had been corrupted the image is getting high; therefore, it is quite difficult for the diffusion to take place smoothly.

The number of iterations also increases with the increase level of noise. The effect on the various numbers of iterations may be due to inconsistency range of pixel distribution of each grayscale image tested. Therefore, the MSSIM also is said to be dependent on the grayscale image properties itself.

The processing time for the program also did not take too long for processing the image as the recorded time is in second (s). It can be observed that most of the time recorded are more than 1 minutes, this is due to properties of ADI scheme that needs to iterate in the two dimensional spaces which are $x$ and $y$ spaces separately. It is quite complicated, but it is worth to be applied since it is faster than the normal implicit algorithm. With just a small gradient threshold parameter, $K=10$ the scheme able to simulate the results for the grayscale image for image denoising. The selection of time step $=0.15$, able to remove the image noise and gives high MSSIM value. All in all, it has been proven that the DG-ADI scheme able to remove the noise with higher density level.

\section{Conclusion}

This paper illustrates on how the DG-ADI scheme works for different level of noise in image denoising. Based on the experiment on the grayscale image, the ADI scheme able to remove noise with different level of noise without disrupting the structural information too much and the edges are preserved well. The discretised schemes are successfully implemented for five different corrupted grayscale images that had been evaluated based on the MSSIM, PSNR UIQI and CPU elapsed time. Hence, it can be concluded that the unconditional stable ADI scheme able to remove noise in higher level of noise even though the image does not fully filtered, but it can prevent from high risks of blurring effect at a specific location based on the MSSIM index values.

\section{Acknowledgement}

The authors would like to express our gratitude to Faculty of Computer and Mathematical Sciences, Universiti Teknologi MARA, Shah Alam for the opportunity and technical support in completing this research.

\section{References}

[1] Chuah JH, Khaw HY, Soon FC \& Chow C (2017), Detection of Gaussian Noise and Its Level using Deep Convolutional Neural Network. Proceedings of the IEEE Region 10 Conference, 24472450.

[2] Farooque MA \& Rohankar JS (2013), Survey on Various Noises and Techniques for Denoising the Color Image. International Journal of Application or Innovation in Engineering and Management 2(11), 217-221.

[3] Guo Z, Sun J, Zhang D \& Wu B (2012), Adaptive Perona-Malik Model based on the Variable Exponent for Image Denoising. IEEE Transactions on Image Processing 21(3), 958-967.

[4] Halim SA, Razak RA, Ibrahim A \& Manurung YH (2014), Perona Malik Anisotropic Diffusion Model using Peaceman Rachford Scheme on Digital Radiographic Image. AIP Conference Proceedings 1602(1), 208-214.

[5] Isogawa K, Ida T, Shiodera T \& Takeguchi T (2018), Deep Shrinkage Convolutional Neural Network for Adaptive Noise Reduction. IEEE Signal Processing Letters 25(2), 224-228.

[6] Kamalaveni V, Rajalakshmi RA \& Narayanankutty KA (2015), Image Denoising Using Variations of Perona-Malik Model with Different Edge Stopping Functions. Procedia Computer Science 58 673-682.

[7] Kaur S (2015), Noise Types and Various Removal Techniques. International Journal of Advanced Research in Electronics and Communication Engineering 4(2), 226-230.

[8] Li S, Zhang D \& Wu B (2011). A New Anisotropic Diffusion Model for Image Denoising. Proceedings of the International Conference on Multimedia Technology, pp. 5707-5710.

[9] McDonough J (2007), Lectures on Computational Numerical Analysis of Partial Differential Equations, Lecture Notes. http://pdf-release.net/external/1997378/pdf-release-dot-net-me690lctr-nts.pdf.

[10] Mihcak MK (1999), Low-complexity Image Denoising based on Statistical Modeling of Wavelet Coefficients. IEEE Signal Processing Letters 6(12), 300-303.

[11] Ostojic V, Starcevic D \& Petrovic V (2016), Recursive Anisotropic Diffusion Denoising. Electronics Letters 2(17), 1449-1451.

[12] Patidar P, Gupya M, Sirvastava S \& Nagawat AK (2010), Image De-noising by Various Filters for Different Noise. International Journal of Computer Applications 9(4), 45-50.

[13] Perona P \& Malik J (1990), Scale-space and Edge Detection using Anisotropic Diffusion. IEEE Transactions on Pattern Analysis and Machine Intelligence 12(7), 629-639.

[14] Tsiotsios C \& Petrou M (2013), On the Choice of the Parameters for Anisotropic Diffusion in Image Processing. Pattern Recognition 46(5), 1369-1381.

[15] Weickert J (1998), Anisotropic Diffusion in Image Processing. B. G. Teubner (Stuttgart), pp.15-25.

[16] Witkin AP (1983), Scale-space Filtering. Proceedings of the International Joint Conference on Artificial Intelligence, pp. 10191022.

[17] Yu J, Zhai R \& Yie J (2017), Image Denoising Method Based on PM Model with Transforming Edge Stopping Function. Proceedings of the 2017 IEEE 2nd Inernational Information Technology, Networking, Electronic and Automation Control Conference, pp. 438-442. 